\title{
Concurrent Infection with Plasmodium vivax and the Dengue and Chikungunya Viruses in a Paediatric Patient from New Delhi, India in 2016
}

\author{
Ayesha Tazeen $^{a}$ Mohd Abdullah ${ }^{a, c}$ Malik Hisamuddin ${ }^{a, c}$ Sher Ali ${ }^{a}$

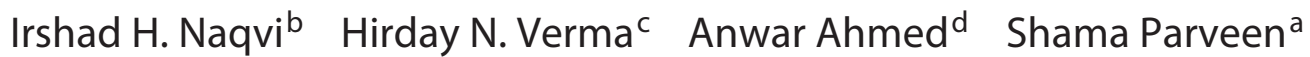 \\ ${ }^{a}$ Centre for Interdisciplinary Research in Basic Sciences and b Dr. M.A. Ansari Health Centre, Jamia Millia Islamia,

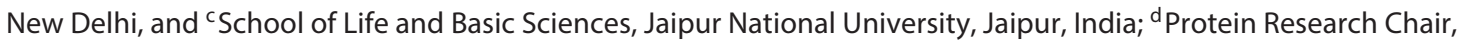 \\ Department of Biochemistry, College of Science, King Saud University, Riyadh, Saudi Arabia
}

\section{Keywords}

Dengue virus - Chikungunya fever - Plasmodium vivax · Concurrent infection

\begin{abstract}
Dengue and chikungunya fevers are transmitted by the common mosquito vector Aedes and malaria by Anopheles. Concurrent infections are reported due to co-circulation of these pathogens, especially in endemic regions. We report a rare case of triple infection with 3 arthropod-borne pathogens (Plasmodium vivax and the dengue and chikungunya viruses) in a 3-year-old child from New Delhi, India, in August 2016. The viruses were identified by RT-PCR and the parasite by microscopy and antigen detection. The dengue virus serotype 3 sequence was clustered in the genotype III by the phylogenetic analysis. Mixed infection with multiple pathogens is a challenge for accurate diagnosis due to the overlapping clinical symptoms. The accurate and timely diagnosis of multiple pathogens in such cases is important for rapid and effective patient management.

(c) 2017 S. Karger AG, Basel
\end{abstract}

\section{Introduction}

Arthropod-mediated infections have become a global health concern, especially in tropical and sub-tropical regions. Dengue and chikungunya fever, caused by the dengue virus (DENV) and the chikungunya virus (CHIKV), respectively, are transmitted by the same mosquito vector, Aedes, leading to their co-circulation in a region [1]. Malaria, caused by the protozoa Plasmodium vivax, $P$. falciparum, P. ovale, and P. malariae, is transmitted among humans by the Anopheles mosquito. There are reports of co-infections with DENV and CHIKV in different geographical regions [1]. Earlier studies also reported dual infections with Plasmodium and DENV/CHIKV [2-6], but concurrent infections with Plasmodium, DENV, and CHIKV are usually not reported in the literature [7]. Furthermore, such triple infections are likely to escape attention due to limited diagnostic facilities, especially in developing countries. Significantly, all 3 of these arthropodmediated infections have overlapping clinical symptoms. A differential diagnosis of the exact aetiological agent of

Ayesha Tazeen and Mohd Abdullah contributed equally to this work.

Dr. Shama Parveen

Centre for Interdisciplinary Research in Basic Sciences Jamia Millia Islamia

New Delhi 110025 (India)

E-Mail shamp25@yahoo.com or sparveen2@jmi.ac.in 
Table 1. Details of the laboratory investigation

\begin{tabular}{ll}
\hline Test & Results \\
\hline Haemoglobin & $7.7 \mathrm{~g} / \mathrm{dL}$ \\
Total leukocyte count & $6,600 \mathrm{cells} / \mathrm{mm}^{3}$ \\
Red blood cell count & $3.54 \times 10^{12} / \mathrm{lacs}$ \\
Haematocrit & $27.7 \%$ \\
Mean corpuscular volume & $78.3 \mathrm{fL}$ \\
Mean corpuscular haemoglobin concentration & $27.8 \mathrm{~g} / \mathrm{dL}$ \\
Mean corpuscular haemoglobin & $21.8 \mathrm{pg} / \mathrm{cell}$ \\
Differential leukocyte count & \\
$\quad$ Polymorphs & $43 \%$ \\
$\quad$ Leukocytes & $49 \%$ \\
$\quad$ Monocytes & $2 \%$ \\
$\quad$ Eosinophils & $7 \%$ \\
Platelet count & $1.15 \mathrm{lacs} / \mathrm{mm}^{3}$ \\
Malaria parasite (thin smear) & positive \\
Parasitemia & $2.36 \%$ \\
Malaria parasite (antigen-based) & positive \\
Dengue virus IgM (ELISA) & negative \\
Dengue virus (RT-PCR) & positive \\
Chikungunya virus IgM (ELISA) & negative \\
Chikungunya virus (RT-PCR) & positive \\
\hline
\end{tabular}

the fever in such cases is very essential for rapid and effective patient management.

New Delhi, the capital of India, reported a re-emergence of dengue and chikungunya fever-like illnesses in August to October 2016 [8]. Torrential rains, high temperatures, rampant construction, and inadequate drainage facilities provided favourable conditions for the proliferation of mosquitoes in Delhi during 2016. This report from New Delhi, India, describes a rare paediatric case of mixed infection caused by Plasmodium, DENV, and CHIKV during the monsoon season of 2016.

\section{Case Report}

A 3-year-old male child living in Jamia Nagar, New Delhi, India, attended the Paediatric Out-Patient Department (OPD) of the Dr. M.A. Ansari Health Centre at the nearby Jamia Millia Islamia campus on the 24th August 2016. This primary health care centre provides basic medical facilities to around 70,000 patients, consisting of students and employees at the University and their dependants. The Health Centre has a full time paediatrician. Usually, patients with mild fever or other mild clinical symptoms visit the Health Centre as it is a small facility available on campus; more severe patients visit the tertiary hospital situated in the adjoining neighborhood.

The clinical history of the patient included a high-grade fever $\left(40^{\circ} \mathrm{C}\right)$ for the last $5-7$ days, along with other symptoms includ- ing chills, uneasiness, body pain, and headache. The patient also showed excessive weakness, abdominal discomfort, and a pale facial appearance. He had no rashes or haemorrhagic tendencies during the OPD visit and there had been no other infections in any of his family member in the last week. An acute-phase blood sample was collected from the patient for the laboratory investigation as advised by the on-duty paediatrician. This sample was collected during an ongoing investigation involving the characterization of the dengue and chikungunya viruses in our laboratory. The investigation was approved by the Institutional Ethics Committee of Jamia Millia Islamia. Written informed consent was obtained from the parents of the patient in English and Hindi.

The initial laboratory investigations included a haemogram, malaria antigen testing, a peripheral smear for malaria parasites, and IgM ELISA for the detection of dengue and chikungunya viruses. The blood investigation report showed low blood indices (Table 1). The peripheral blood smear revealed microcytic hypochoromic anaemia and positive for the haemoparasite $P$. vivax at the schizont stage. The blood smear was repeated to rule out any possibility of a mixed parasite infection with $P$. falciparum, but it showed only $P$. vivax. The presence of $P$. vivax was later confirmed by antigen detection kit. The serum sample was negative for dengue and chikungunya IgM antibodies by ELISA. The reports of the all the abovementioned laboratory investigations were provided to the patient on the same day, and he was diagnosed as having malaria on the basis of the initial clinical symptoms and laboratory investigations. He was prescribed symptomatic treatment with the recommended paediatric dose of chloroquine along with paracetamol and an antacid at the Health Centre. He was not sick enough for hospitalization. We tried to contact the parents of the patient for follow-up, but they were not available. Later on, we were informed that the patient went back to his home town with his parents, and so the follow-up and treatment outcome were not recorded.

Within the next 4 days, the sample was tested for DENV and CHIKV by RT-PCR at the virology laboratory. The sample was collected during the monsoon period of 2016, which coincided with a large number of reported DENV/CHIKV cases in Delhi [8]. RT-PCR was performed on the serum sample collected from the patient using published primers $[9,10]$ utilizing the conditions that have been standardized in our laboratory [11]. The sample was found to be positive for both the viruses by RT-PCR. The PCR reactions were carried out with appropriate positive and negative controls to exclude contamination or false-positive findings. The 292-bp amplicon specific for the DENV serotype 3 (DENV-3) CprM region and the 852-bp amplicon specific for the CHIKV envelope gene were obtained from the sample (Fig. 1). The presence of both viruses was confirmed by performing RT-PCR using 2 independent RNA extraction procedures. However, the CHIKV strain could not be sequenced due to the limited volume of the clinical sample. The presence of the DENV strain was further confirmed by commercial DNA sequencing of the partial CprM region (GenBank accession No. KY099620). The DENV sequence was confirmed by the BLAST algorithm and was edited manually in GeneDoc v2.7 and BioEdit v7.2.5 software. The study sequence and the GenBank sequence of DENV-3 were aligned, and phylogenetic trees were constructed in MEGA v6.0 using the maximum likelihood method (Fig. 2). Phylogenetic analysis clustered the study DENV-3 sequence (292-bp) within the genotype III, close to the sequences from India, Cuba, Martinique, and Paraguay. 
Fig. 1. Agarose gel photographs show the detection of CHIKV (a, 852-bp amplicon) and DENV serotype 3 (b, 292-bp amplicon) from a clinical sample. Lane 1, 100bp ladder; lane 2, CHIKV/DENV-specific amplicon. CHIKV, chikungunya virus; DENV, dengue virus.
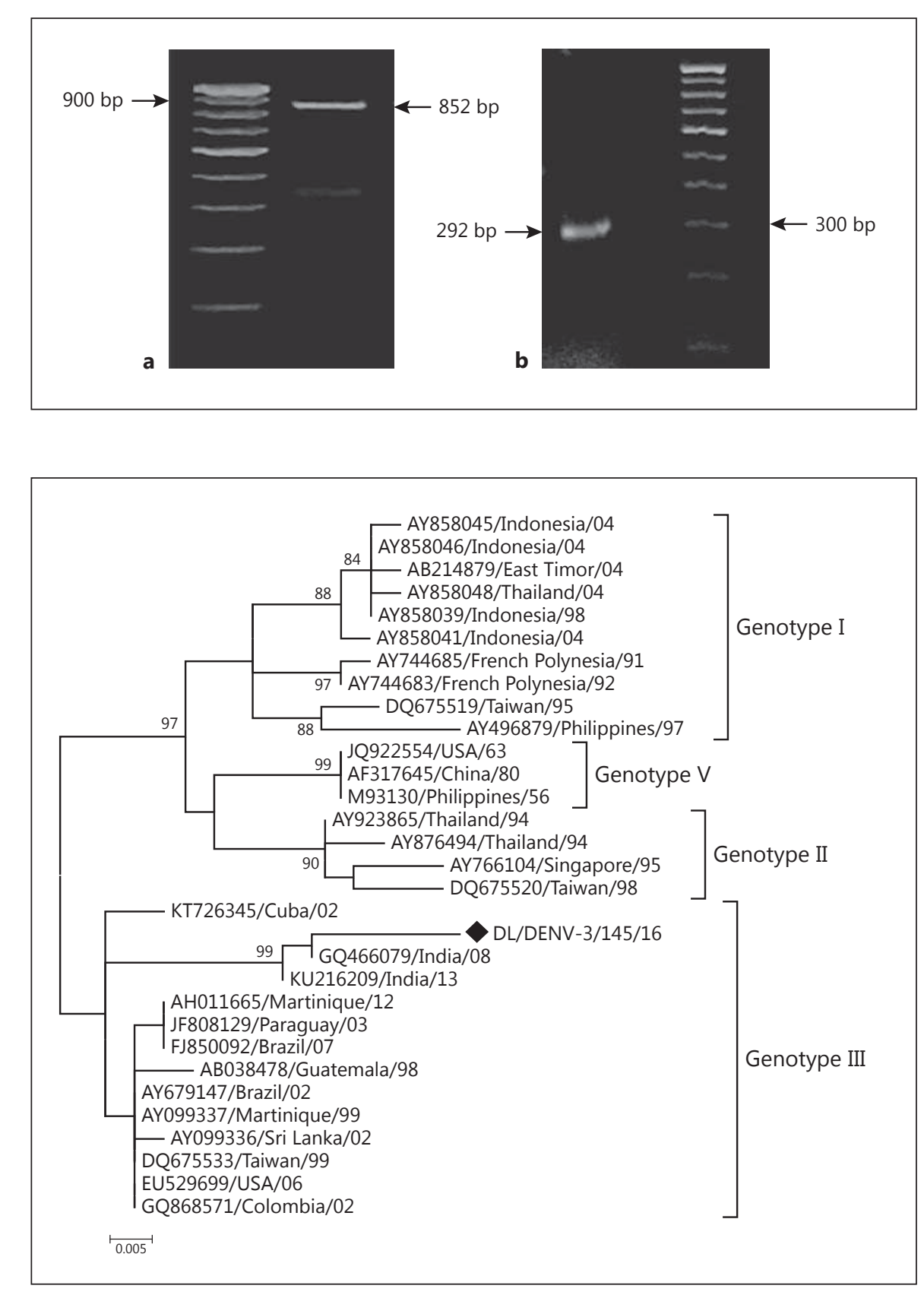

Fig. 2. Maximum likelihood phylogenetic tree of dengue virus serotype 3 based on the partial CprM region. The tree was constructed based on the Tamura-Nei model. Bootstrap values are represented by the numbers on nodes generated by 1,000 replications. The study sequence $(\bullet)$ was clustered within genotype III.

\section{Discussion}

This investigation describes the rare case of a paediatric patient who showed concurrent infection with DENV, $\mathrm{CHIKV}$, and P. vivax. This report is from Jamia Millia Islamia, situated adjacent to bank of the Yamuna River in the south-eastern part of New Delhi, India. This region has high humidity, high temperatures, and a dense population, and along with poor sanitation, these conditions are conducive to mosquito proliferation. All these arthropod-mediated infections are transmitted by mosquitoes. Appropriate precautions should be taken to prevent the transmission of these infections. Prevention of mosquito breeding in stagnant water during the monsoon season and the use of mosquito nets, repellent creams, and insecticidal sprays have been proven to be effective in controlling the mosquito population, especially during epidemics. Furthermore, generating awareness about different
Tazeen/Abdullah/Hisamuddin/Ali/Naqvi/ Verma/Ahmed/Parveen 
aspects of the infections and their transmission will also contribute towards better management of these diseases.

Several investigations have reported concurrent infections with DENV and CHIKV in different geographical regions including India [1,11-14]. Previous studies have described dual infections of Plasmodium and other arboviruses including DENV or CHIKV [2-4]. But co-infections with 3 different pathogens are rarely reported in the literature. In a recent report from Bengaluru, India, a triple infection with CHIKV, DENV, and malaria was described in a traveller in 2015 [7]. However, to the best of our knowledge, this is the first case report of such triple infection in New Delhi, India, in 2016.

Mixed infections causing more than one mosquitoborne illness like malaria and dengue and chikungunya fevers pose a challenge for the accurate diagnosis of the aetiological agents due to overlapping clinical symptoms. These 3 arthropod-mediated infections have common symptoms including fever, rashes, headache, nausea, vomiting, abdominal pain, myalgia, and arthralgia. It is therefore difficult to differentiate these 3 infections on the basis of the clinical condition alone. A previous study showed that fever may be prolonged in cases of concurrent infections [15]. Thus, laboratory diagnosis along with the clinical symptoms might assist the clinicians in making an accurate diagnosis. Such concurrent infections may further complicate management, especially in paediatric patients. It has been previously shown that a dengue and malaria co-infection requires special attention because a delayed diagnosis and inappropriate treatment can result in fatal complications $[16,17]$ or an aggravated host immune response [18]. In some patients, concurrent infections are more severe than mono-infec- tions $[15,18]$; others show no difference between co-infections and isolated infections [19]. Thus, an appropriate, differential, and timely diagnosis of pathogens and the correlation with disease severity of mono- versus coinfections are important for effective patient management. While the consequences of this study on simultaneous infection by multiple pathogens were not established, we recommend our method for future reference in the context of epidemiological investigations. Correlations of the clinical, epidemiological, immunological, and genetic basis of concurrent infections should be incorporated in future investigations. It is envisaged that working along these lines will uncover the incidence and likely molecular mechanisms of co-infection and the disease burden caused by arthropod-mediated pathogens.

\section{Acknowledgements}

A.T. was supported by Research Fellowship of the University Grants Commission, Government of India and M.H. by a Senior Research Fellowship of Indian Council of Medical Research, New Delhi, India. S.A. acknowledges the award of a J.C. Bose National Fellowship from the DST-SERB, New Delhi, India.

Work in our laboratory is supported by grants from the University Grants Commission and the Council of Scientific and Industrial Research, Government of India. We acknowledge the support of the laboratory staff of Dr. M.A. Ansari Health Centre, Jamia Millia Islamia, New Delhi, India. This study was supported by the King Saud University, Vice Dean of Research Chairs.

\section{Disclosure Statement}

The authors declare that they have no competing interests.

\section{References}

1 Deeba F, Afreen N, Islam A, et al: Co-infection with dengue and chikungunya viruses; in: Current Topics in Chikungunya, chapt 6 . Croatia, INTECH open science, 2016.

2 Magalhães BM, Siqueira AM, Alexandre MA, Souza MS, Gimaque JB, Bastos MS, et al: $P$. vivax malaria and dengue fever co-infection: a cross-sectional study in the Brazilian Amazon. PLoS Negl Trop Dis 2014;8:e3239.

3 Carme B, Matheus S, Donutil G, Raulin O, Nacher M, Morvan J: Concurrent dengue and malaria in Cayenne Hospital, French Guiana. Emerg Infect Dis 2009;15:668-671.

4 Ayorinde AF, Oyeyiga AM, Nosegbe NO, Folarin OA: A survey of malaria and some arboviral infections among suspected febrile pa- tients visiting a health centre in Simawa, Ogun State, Nigeria. J Infect Public Health 2016;9:52-59.

5 Thangaratham P, Jeevan M, Rajendran R, Samuel PP, Tyagi B: Dual infection by dengue virus and Plasmodium vivax in Alappuzha District, Kerala, India. Jpn J Infect Dis 2006;59:211.

6 Chong SE, Zaini RHM, Suraiya S, Lee KT, Lim JA: The dangers of accepting a single diagnosis: case report of concurrent Plasmodium knowlesi malaria and dengue infection. Malar J 2017;16:2.

7 Raut C, Rao N, Sinha D, Hanumaiah H, Manjunatha M: Chikungunya, dengue, and malaria co-infection after travel to Nigeria, India. Emerg Infect Dis 2015;21:908-909.
8 Nazish P, Broor S, Abdullah M, Malik H, Tazeen A, Asif N, et al: Resurgence of denguelike and chikungunya-like illness in New Delhi, India, during 2016. Indian J Health Sci 2016;3:61-65.

9 Lanciotti RS, Calisher CH, Gubler DJ, Chang G-J, Vorndam AV: Rapid detection and typing of dengue viruses from clinical samples by using reverse transcriptase-polymerase chain reaction. J Clin Microbiol 1992;30: 545-551.

10 Santhosh S, Dash P, Parida M, Khan M, Tiwari M, Rao PL: Comparative full genome analysis revealed E1:A226V shift in $2007 \mathrm{In}$ dian chikungunya virus isolates. Virus Res 2008; $135: 36-41$.
Mixed Infection with P. vivax, DENV, and CHIKV
Intervirology 2017;60:48-52 DOI: $10.1159 / 000479430$ 
11 Afreen N, Deeba F, Khan WH, Haider SH, Kazim SN, Ishrat R, et al: Molecular characterization of dengue and chikungunya virus strains circulating in New Delhi, India. Microbiol Immunol 2014;58:688-696.

12 Edwards T: Co-infections with chikungunya and dengue viruses, Guatemala, 2015. Emerg Infect Dis 2016;22:2003-2005.

13 Furuya-Kanamori L, Liang S, Milinovich G, Magalhaes RJS, Clements AC, $\mathrm{Hu}$ W, et al: Co-distribution and co-infection of chikungunya and dengue viruses. BMC Infect Dis 2016;16:1.
14 Chahar HS, Bharaj P, Dar L, Guleria R, Kabra SK, Broor S: Co-infections with chikungunya virus and dengue virus in Delhi, India. Emerg Infect Dis 2009; 15:1077.

15 Epelboin L, Boullé C, Ouar-Epelboin S, Hanf M, Dussart P, Djossou F, et al: Discriminating malaria from dengue fever in endemic areas: clinical and biological criteria, prognostic score and utility of the C-reactive protein: a retrospective matched-pair study in French Guiana. PLoS Negl Trop Dis 2013;7:e2420.

16 Charrel RN, Brouqui P, Foucault C, de Lamballerie X: Concurrent dengue and malaria. Emerg Infect Dis 2005;11:1153.
17 Ward DI: A case of fatal Plasmodium falciparum malaria complicated by acute dengue fever in East Timor. Am J Trop Med Hyg 2006; $75: 182-185$

18 Mendonça VR, Andrade BB, Souza LC, Magalhães BM, Mourão MP, Lacerda MV, et al: Unravelling the patterns of host immune responses in Plasmodium vivax malaria and dengue co-infection. Malar J 2015; 14:315.

19 Halsey ES, Baldeviano GC, Edgel KA, Vilcarromero S, Sihuincha M, Lescano AG: Symptoms and immune markers in Plasmodium/ dengue virus co-infection compared with mono-infection with either in Peru. PLoS Negl Trop Dis 2016; 10:e0004646. 